\title{
Impact of gestational diabetes on the risk of diabetes following pregnancy among Chinese and South Asian women
}

\author{
G. Mukerji • M. Chiu • B. R. Shah
}

Received: 16 December 2011 /Accepted: 12 March 2012/Published online: 18 April 2012

(C) Springer-Verlag 2012

\begin{abstract}
Aims/hypothesis Ethnicity and gestational diabetes mellitus (GDM) are both risk factors for the development of type 2 diabetes. However, it is uncertain whether ethnicity modifies the effect of GDM on diabetes risk. We aimed to determine the risk of diabetes following pregnancy with and without GDM for Chinese and South Asian women compared with white women.

Methods Using healthcare databases, all 1,050,108 women aged 20-49 with live births between January 1995 and June 2008 in Ontario were identified. They were followed for up to 15 years for the diagnosis of diabetes.

Results The age-standardised prevalences of GDM were $4.1 \%, 7.1 \%$ and $2.9 \%$ for Chinese, South Asian and white women, respectively. The cumulative incidence of diagnosed diabetes at the median follow-up time of 7.6 years was $16.5 \%$ and $1.8 \%$ for Chinese women with and without GDM, 31.8\% and 3.6\% for South Asian women with and without GDM, and $25.7 \%$ and $1.8 \%$ for white women with and without GDM. The presence of GDM conferred an increase in the risk for diabetes after pregnancy of more than 13-fold in white women, but only a nine- to tenfold increase among Chinese and South Asian women.
\end{abstract}

G. Mukerji $\cdot$ M. Chiu $\cdot$ B. R. Shah

University of Toronto,

Toronto, ON, Canada

M. Chiu • B. R. Shah $(\bowtie)$

Institute for Clinical Evaluative Sciences,

G106-2075 Bayview Avenue,

Toronto, ON, Canada M4N 3M5

e-mail: baiju.shah@ices.on.ca

B. R. Shah

Sunnybrook Health Sciences Centre,

Toronto, ON, Canada
Conclusions/interpretation Although one-third of South Asian women with GDM were diagnosed with diabetes within 8 years postpartum, the incremental impact of GDM on diabetes risk was not as strong among Chinese and South Asian women as it was among white women.

Keywords Asian populations · Ethnic groups - Gestational diabetes $\cdot$ Incidence $\cdot$ Type 2 diabetes
Abbreviations
GDM Gestational diabetes mellitus
ODD Ontario Diabetes Database

\section{Introduction}

Gestational diabetes mellitus (GDM) is defined as an abnormal carbohydrate tolerance diagnosed or first recognised in pregnancy, and it affects between $2 \%$ and $7 \%$ of pregnant women $[1,2]$. This antepartum glucose intolerance is a marker of insulin resistance that can persist into the postpartum period [3, 4], and increases the risk of developing type 2 diabetes $[5,6]$.

While ethnicity is another well-recognised risk factor for type 2 diabetes [7-10], less is known about its role in GDM. A 1992 US study showed that white women had a lower prevalence of GDM than all other racial groups [11]. More recent data have corroborated this observation [12-14]. Furthermore, there are data suggesting that women from some minority groups may be at increased risk of glucose intolerance or diabetes following pregnancy complicated by GDM [15-17]. However, these earlier studies were small, and they did not include patients without GDM, so whether the observed ethnic differences in diabetes risk after GDM 
differed from baseline ethnic differences in diabetes risk could not be evaluated. One recent study examined 13,000 Californian women with GDM, and found marked variation between ethnic groups in diabetes incidence, ranging from 15.8 per 1,000 person-years in white women to 29.0 per 1,000 person-years in black women [18]. Notably, this study did not disaggregate Asian populations, despite the known heterogeneity in diabetes risk in Asian subgroups [19, 20].

The two largest minority ethnic groups in Canada are Chinese (people with Chinese, Hong Kong or Taiwanese ancestry) and South Asians (people with Indian [subcontinent] ancestry) [21]. Two-stage universal screening for GDM is recommended for all pregnant women by Canadian clinical practice guidelines [22]. The objective of this study was to determine whether the incremental impact of GDM on the risk for subsequent diabetes after pregnancy differed between Chinese and South Asian women compared with white women. We hypothesised that minority ethnicity and GDM would act synergistically as risk factors for incident diabetes after pregnancy.

\section{Methods}

Study design and data sources We conducted a populationbased cohort study using healthcare databases from the government-funded health insurance programme of the Ontario Ministry of Health and Long-Term Care. This programme is available to all permanent residents of Ontario, Canada's most populous and most ethnically diverse province. These databases include hospital-discharge data, which provide detailed information on all hospitalisations in the province, and the Registered Persons Database, which includes demographic information on all Ontario residents. We also used the Ontario Diabetes Database (ODD), a validated registry of all people diagnosed with non-gestational diabetes in Ontario, derived from healthcare databases [23]. Though the database cannot distinguish between types of diabetes, most incident diabetes in this age group would be of type 2 diabetes. Individuals are linked between these databases and across time by their unique health card number.

Study population All women aged 20-49 years with inhospital live births between 1 January 1995 and 30 June 2008 were identified. For women with multiple deliveries during the study period, one delivery was selected at random as the index delivery. Women with pre-existing diabetes were excluded. Women were defined as having GDM if diabetes was coded on the hospitalisation record for the delivery.

Measured baseline characteristics were age at delivery, socioeconomic status (measured ecologically as the neighbourhood household income, divided into provincewide quintiles), the presence of chronic comorbidities (measured using the Charlson comorbidity index) [24], urban vs rural residence, antenatal ambulatory visits with an obstetrician, and ambulatory visits with a primary care physician in the 2 years preceding delivery (as a baseline measure of intensity of health service use). Women with missing address information, for whom some of these baseline characteristics could not be determined, were excluded.

The cohort was subdivided based on ethnicity. As there are no ethnic identifiers in Canadian healthcare data, assignment of people to Chinese and South Asian ethnic groups was based on their surnames, using two lists of surnames validated to identify people with Chinese and South Asian origins in Ontario [25]. Both lists demonstrate excellent positive predictive values when compared with selfreported ethnicity $(91.9 \%$ for Chinese and $89.3 \%$ for South Asian) [25]. Women whose surnames were not on either list were defined as white, as whites make up approximately $87 \%$ of the non-Chinese/non-South Asian population of Ontario [21].

Outcome of interest and statistical analysis Both crude and age-standardised prevalences of GDM were determined for each population.

The primary outcome of interest was diagnosis of diabetes after pregnancy, determined by entry into the ODD postpartum. All women were followed until 31 March 2010. Cumulative incidence curves were constructed for diagnosed diabetes after pregnancy, stratified by ethnic group and by GDM status. Cox proportional hazards regression models were fitted in which individuals were censored at death: both an unadjusted model and a model adjusting for age, socioeconomic status and comorbidity. The independent variables of interest were GDM status and ethnicity. An interaction term between GDM status and ethnicity was included to test for effect modification. The assumption of proportionality was confirmed for all models by plotting log (- $\log [$ survival] $)$ vs $\log ($ time $)$ to assess parallelism.

All statistical analyses were performed using SAS version 9.2 .

Ethics approval The study was approved by the Research Ethics Board of Sunnybrook Health Sciences Centre, Toronto, ON, Canada.

\section{Results}

There were 1,069,098 women with live births during the study period. Of these, 14,591 (1.4\%) were excluded because of pre-existing diabetes, and a further $4,399(0.4 \%)$ were excluded because of missing address information. The 
baseline characteristics of the final 1,050,108 individuals are shown in Table 1.

GDM occurred in 33,203 (3.2\%) pregnancies. The crude prevalence of GDM was higher for both minority groups: $5.0 \%$ for Chinese and $6.5 \%$ for South Asian compared with $2.9 \%$ for white women. When standardised for age, the prevalence of GDM among Chinese women was attenuated but remained higher than in white women $(4.1 \%$; $95 \%$ CI $3.9 \%, 4.3 \% ; p<0.0001)$, whereas the age-standardised prevalence of GDM among South Asian women was even higher than the crude prevalence $(7.1 \% ; 95 \%$ CI $6.8 \%$, $7.4 \% ; p<0.0001$ vs white women).

Diabetes was diagnosed in 30,234 of the women in the cohort: 7,755 of the women with GDM at index (39.8 cases per 1,000 person-years), and 22,479 of the women without GDM (2.8 cases per 1,000 person-years). Incidence rates were $2.7,5.3$ and 2.7 cases per 1,000 person-years for Chinese, South Asian and white women without GDM, respectively, and 25.8, 54.7 and 39.7 cases per 1,000 person-years for Chinese, South Asian and white women with GDM, respectively. The cumulative incidence of diagnosed diabetes after pregnancy is shown in Fig. 1. At the median follow-up time of 7.6 years, $1.8 \%$ of Chinese women, $3.6 \%$ of South Asian women and $1.8 \%$ of white women without GDM were diagnosed with diabetes, compared with $16.5 \%$ of Chinese women, $31.8 \%$ of South Asian women and $25.7 \%$ of white women with GDM.

Both GDM status and ethnicity were independently associated with diabetes diagnosis in the Cox proportional hazard models, and a statistically significant interaction term

Table 1 Baseline characteristics of women delivering between 1995 and 2008

\begin{tabular}{llll}
\hline Characteristic & $\begin{array}{l}\text { Chinese } \\
n=49,607\end{array}$ & $\begin{array}{l}\text { South Asian } \\
n=42,521\end{array}$ & $\begin{array}{l}\text { White } \\
n=958,980\end{array}$ \\
\hline $\begin{array}{l}\text { Median age } \\
\text { (interquartile range) }\end{array}$ & $32(29-35)$ & $29(26-32)$ & $30(26-34)$ \\
$\begin{array}{l}\text { Socioeconomic status } \\
\text { Lowest }\end{array}$ & 26 & 32 & \\
2 & 26 & 26 & 22 \\
3 & 19 & 21 & 20 \\
4 & 17 & 14 & 21 \\
$\quad$ Highest & 12 & 7 & 17 \\
Chronic comorbidity & 0.6 & 0.3 & 0.8 \\
Rural residence & 0.6 & 0.3 & 11.0 \\
Antenatal obstetrician & 92.9 & 93.2 & 82.2 \\
$\quad$ care & & & \\
Number of primary & & & \\
$\quad$ care visits & & 65.5 & 57.8 \\
$\geq 10$ & 44.9 & 34.5 & 42.2 \\
$\quad<10$ & 55.1 & & \\
\hline
\end{tabular}

Results are presented as \%, unless stated otherwise between the two indicated that effect modification was present. Table 2 shows the HRs and 95\% CIs for specific comparisons of interest from both the unadjusted and adjusted models. Compared with white women with GDM, Chinese women with GDM were at decreased risk of being diagnosed with diabetes, whereas South Asian women with GDM were at increased risk. When compared with their counterparts without GDM, white women with GDM were at greater risk of being diagnosed with diabetes (adjusted HR 13.6; 95\% CI 13.2, 14.0) than either Chinese women with GDM (adjusted HR 9.2; 95\% CI 8.1, 10.3) or South Asian women with GDM (adjusted HR 9.6; 95\% CI 8.8, 10.5).

\section{Discussion}

In this large population-based study of over 1 million women followed for up to 15 years, we observed ethnic differences in the prevalence of GDM and in the incremental impact of GDM on the risk of diabetes after pregnancy. The age-adjusted prevalence of GDM was $41 \%$ higher among Chinese and 145\% higher among South Asians compared with white women. Among women with GDM, incident diabetes increased most rapidly in the first 4 to 6 months postpartum, with many of these cases probably representing previously undiagnosed type 2 diabetes that was first recognised during screening in pregnancy. Of note, previous data have shown that undiagnosed diabetes is not associated with minority ethnicity in Canada [26], so it is unlikely to bias the results. South Asians with GDM had the greatest risk of being subsequently diagnosed with diabetes, with a cumulative incidence of $50 \%$ by 12.5 years postpartum. In contrast, despite having a higher prevalence of GDM than white women, Chinese women with GDM had a lower risk of being diagnosed with diabetes after pregnancy, suggesting that the metabolic abnormalities and insulin resistance leading to GDM in Chinese women are unique to pregnancy and do not persist postpartum to the same degree as in white women. In fact, notwithstanding the differences in the absolute risk, the relative incremental impact of GDM on future diabetes risk was lower among both minority groups than in white women: the presence of GDM in the index pregnancy led to a $>13$-fold increase in the risk of a subsequent diagnosis of diabetes for white women, but conferred only a nine- to tenfold increase in risk for both Chinese and South Asian women.

This study confirms the results of two prior populationbased studies that showed that Chinese and South Asian women have a higher prevalence of GDM than white women. A large Australian study demonstrated that women born in East or South Asia had an increased risk of developing GDM relative to women born in Australia (ORs 3.2 and 4.2, 
Fig. 1 Cumulative incidence of diabetes diagnosis after pregnancy, stratified by ethnicity and GDM status

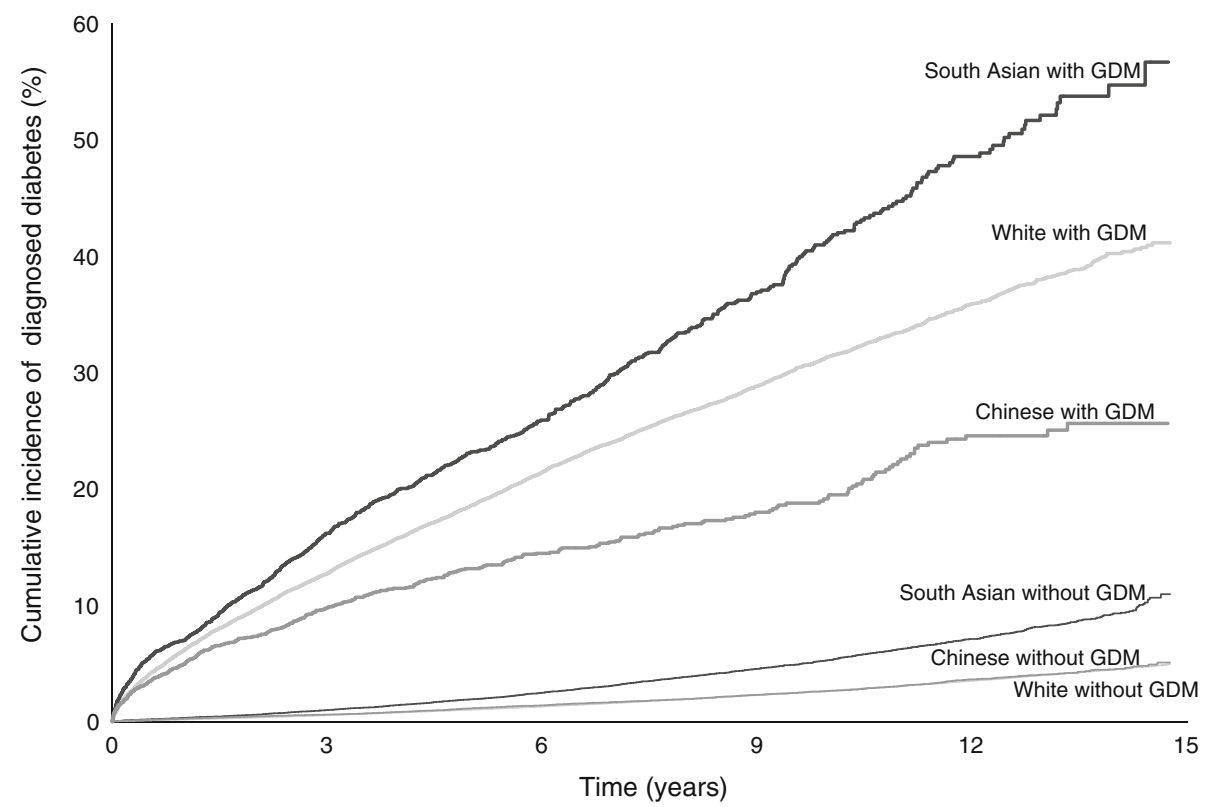

respectively) [27]. Similarly, a smaller study in New York City demonstrated an increased risk of GDM among virtually all minority populations compared with whites, including an adjusted relative risk of 2.3 for Chinese women and 4.7 for South Asian women [28]. The high risk of subsequent diabetes after GDM among South Asians has been previously described in a small UK study, where the cumulative diabetes incidence at 4.4 years after pregnancy was 49\% among South Asians but only 25\% among whites [17]. Other studies have similarly described ethnic variation in the risk of subsequent diabetes after GDM [6, 18, 29], but have not shown effect modification of Asian ethnicities on the relationship between GDM and subsequent diabetes risk.

Rather than ethnicity and GDM acting synergistically on the risk of subsequent diabetes after pregnancy as hypothesised, minority ethnicity actually attenuated the impact of GDM on future diabetes risk. Hence, the relative impact of GDM on diabetes risk compared with the absence of GDM was not as strong for Chinese and South Asian women as it was for white women. The possible explanations for this observation and the greater prevalence of GDM among minority women are uncertain, but lifestyle, dietary or cultural factors may contribute. Alternatively, Chinese and
South Asian women may have metabolic differences from white women that reflect higher degrees of antenatal insulin resistance and therefore relatively greater degrees of glucose intolerance in pregnancy $[17,28,30]$, but that do not necessarily lead to similar degrees of insulin resistance postpartum. The original diagnostic thresholds for GDM described by O'Sullivan, which were shown to predict postpartum diabetes incidence, were derived from a predominantly white population in New England [31]. Our study suggests that it may be inappropriate to generalise these thresholds to other ethnic groups, where antenatal glucose distributions and their relationship to future diabetes risk may be different. A recent Canadian study supported the hypothesis that normative glucose distributions during pregnancy may be higher among minority populations compared with white women [32].

Our study has several strengths to note: it is by far the largest and longest duration study to report on the risk of incident diabetes following GDM in any population. In addition, the use of secondary healthcare data ensured that the data were comprehensive, complete and truly population-based. However, a number of limitations deserve mention. First, the secondary data sources had limited
Table 2 Comparisons between selected groups for the risk of diabetes diagnosis after pregnancy

\footnotetext{
${ }^{a}$ Adjusted for age, socioeconomic status and comorbidity
}

\begin{tabular}{lllll}
\hline Groups compared & & & \multicolumn{2}{l}{ HR $(95 \% \mathrm{CI})$} \\
\cline { 1 - 1 } \cline { 5 - 5 } Group 1 & Group 2 & & Unadjusted & Adjusted $^{\mathrm{a}}$ \\
\hline Chinese with GDM & White with GDM & & $0.7(0.6,0.7)$ & $0.6(0.6,0.7)$ \\
South Asian with GDM & White with GDM & & $1.4(1.3,1.5)$ & $1.4(1.3,1.5)$ \\
Chinese with GDM & Chinese without GDM & & $9.8(8.7,11.1)$ & $9.2(8.1,10.3)$ \\
South Asian with GDM & South Asian without GDM & & $10.8(9.9,11.8)$ & $9.6(8.8,10.5)$ \\
White with GDM & White without GDM & & $15.3(14.9,15.7)$ & $13.6(13.2,14.0)$ \\
\hline
\end{tabular}


ascertainment of clinical details that may be associated with diabetes risk (such as pre-pregnancy obesity, weight gain during pregnancy or glycaemic control). However, ethnic differences in many of these risk factors may form part of the causal pathway for differences in diabetes risk, so adjusting for them would be inappropriate. Although adjusting for pre-pregnancy obesity would have been optimal had these data existed, a previous study examining diabetes risk after GDM found that such adjustment did not materially influence differences between ethnicities [18]. Second, we did not have access to patients' actual glucose levels, and we were not able to verify that postpartum diabetes testing was complete, so differences in screening rates could affect the observed results. Instead, the study relied on a populationlevel health services approach using claims data to identify diagnosed diabetes only. However, undiagnosed diabetes is no more common in high-risk ethnic groups than in other populations in Canada [26], and minority populations actually receive more diabetes screening tests [33], so it is unlikely that ethnic differences in diabetes screening rates influence the observed results. Third, we could not distinguish types of incident diabetes with the available data, and inclusion of type 1 diabetes might elevate the apparent incident diabetes rate for white women compared with Asian women. However, $88 \%$ of the diabetes diagnoses during follow-up occurred when women were aged 30 or older, and studies using claims data commonly use 30 as a threshold to separate type 1 from type 2 diabetes [34, 35]. We re-analysed the data without counting as incident cases the 3,761 women who were diagnosed with diabetes when younger than 30 , and found the adjusted HRs were virtually unchanged. Fourth, the limitations of using surname lists to assign ethnicity include misclassification of people whose surnames do not reflect their ethnicity (e.g. adopting a new surname after marriage). However, these misclassifications would tend to bias the study results towards an absence of differences between ethnic groups. Finally, this study examined only women with Chinese and South Asian ancestries. Women from other ethnic groups who may be at increased risk for GDM and subsequent incident diabetes, including AfroCaribbean and Aboriginal women, share many of their surnames with the white population in Canada, making surnames unsuitable for identification of these ethnic groups. These populations were instead included in the white population in this study, which may have led to an overestimation of diabetes incidence among white women. However, given that these other minority populations made up only $12.9 \%$ of this group, the magnitude of this overestimation is minimal.

In summary, our study showed that both Chinese and South Asian women have a higher prevalence of GDM than white women, which would therefore increase their risk of adverse maternal and neonatal outcomes. Clinicians must ensure that women from these and other high-risk ethnic groups are targeted for GDM screening in pregnancy and that they maintain adequate antenatal blood glucose control. Ethnic-specific healthcare services, such as dietary recommendations using culturally relevant foods, may be required to optimise outcomes. Moreover, there is some evidence that minority women underuse antenatal care despite the publicly funded healthcare system in Canada [36], so policies and interventions to improve access to and use of healthcare services by minority women may be needed to reduce the risk of complications. The study also demonstrated that South Asian women with GDM are at a very high risk of developing diabetes postpartum. However, because South Asian women even without GDM have a higher risk of diabetes than women from other ethnicities, the presence of GDM does not portend as much of a relative increase in the risk of diabetes for South Asian women (and for Chinese women) as it does for white women. The underlying physiological explanation for this observation is uncertain, so a greater understanding of ethnic variations in glucose metabolism during and after pregnancy is needed. Ethnic-specific cut-offs for body mass index and waist circumference have already been proposed to predict long-term metabolic abnormalities [37-39]. This study suggests that ethnic-specific thresholds for the diagnosis of GDM may be needed to more accurately reflect differences in the risk of diabetes and to help identify those patients requiring more intensive followup and intervention postpartum.

Acknowledgements The authors would like to thank F. Wu for assistance with the statistical analysis of the data, and P. Austin and D. Redelmeier for their comments on earlier drafts of the manuscript.

Funding B. R. Shah is supported by the Canadian Institutes of Health Research and the Canadian Diabetes Association. The Institute for Clinical Evaluative Sciences is funded, in part, by the Ontario Ministry of Health and Long-Term Care.

Duality of interest statement The authors declare that there is no duality of interest associated with this manuscript.

Contribution statement BRS and GM participated in the conception and design of the study, and drafted the article. All authors contributed to the analysis and interpretation of data, and the revision of the manuscript for important intellectual content. All authors gave final approval of the version to be published.

\section{References}

1. Jovanovic L, Pettitt DJ (2001) Gestational diabetes mellitus. JAMA 286:2516-2518

2. Ferrara A (2007) Increasing prevalence of gestational diabetes mellitus. Diabetes Care 30:S141-S146 
3. Catalano PM, Huston L, Amini SB, Kalhan SC (1999) Longitudinal changes in glucose metabolism during pregnancy in obese women with normal glucose tolerance and gestational diabetes mellitus. Am J Obstet Gynecol 180:903-916

4. Homko C, Sivan E, Chen X, Reece EA, Boden G (2001) Insulin secretion during and after pregnancy in patients with gestational diabetes mellitus. J Clin Endocrinol Metab 86:568-573

5. Feig DS, Zinman B, Wang X, Hux JE (2008) Risk of development of diabetes mellitus after diagnosis of gestational diabetes. CMAJ 179:229-234

6. Kim C, Newton KM, Knopp RH (2002) Gestational diabetes and the incidence of type 2 diabetes: a systematic review. Diabetes Care 25:1862-1868

7. Wild S, Roglic G, Green A, Sicree R, King H (2004) Global prevalence of diabetes: estimates for the year 2000 and projections for 2030. Diabetes Care 27:1047-1053

8. Anand SS, Yusuf S, Vuksan V et al (2000) Differences in risk factors, atherosclerosis, and cardiovascular disease between ethnic groups in Canada: the Study of Health Assessment and Risk in Ethnic groups (SHARE). Lancet 356:279-284

9. Narayan KMV, Boyle JP, Thompson TJ, Sorensen SW, Williamson DF (2003) Lifetime risk for diabetes mellitus in the United States. JAMA 290:1884-1890

10. Creatore MI, Moineddin R, Booth G et al (2010) Age- and sexrelated prevalence of diabetes mellitus among immigrants to Ontario, Canada. CMAJ 182:781-789

11. Berkowitz GS, Lapinski RH, Wein R, Lee D (1992) Race/ethnicity and other risk factors for gestational diabetes. Am J Epidemiol 135:965-973

12. Ferrara A, Kahn HS, Quesenberry CP, Riley C, Hedderson MM (2004) An increase in the incidence of gestational diabetes mellitus: Northern California, 1991-2000. Obstet Gynecol 103:526-533

13. Lawrence JM, Contreras R, Chen W, Sacks DA (2008) Trends in the prevalence of preexisting diabetes and gestational diabetes mellitus among a racially/ethnically diverse population of pregnant women, 1999-2005. Diabetes Care 31:899-904

14. Hedderson MM, Darbinian JA, Ferrara A (2010) Disparities in the risk of gestational diabetes by race-ethnicity and country of birth. Paediatr Perinat Epidemiol 24:441-448

15. Sinha B, Brydon P, Taylor RS et al (2003) Maternal ante-natal parameters as predictors of persistent postnatal glucose intolerance: a comparative study between Afro-Caribbeans, Asians and Caucasians. Diabet Med 20:382-386

16. Lee AJ, Hiscock RJ, Wein P, Walker SP, Permezel M (2007) Gestational diabetes mellitus: clinical predictors and long-term risk of developing type 2 diabetes. Diabetes Care 30:878-883

17. Oldfield MD, Donley P, Walwyn L, Scudamore I, Gregory R (2007) Long term prognosis of women with gestational diabetes in a multiethnic population. Postgrad Med J 83:426-430

18. Xiang AH, Li BH, Black MH et al (2011) Racial and ethnic disparities in diabetes risk after gestational diabetes. Diabetologia 54:3016-3021

19. Kanaya AM, Adler N, Moffet HH et al (2011) Heterogeneity of diabetes outcomes among Asians and Pacific Islanders in the U.S. Diabetes Care 34:930-937

20. Chiu M, Austin PC, Manuel DG, Shah BR, Tu JV (2011) Deriving ethnic-specific BMI cutoff points for assessing diabetes risk. Diabetes Care 34:1741-1748
21. Statistics Canada. (2008) Ethnocultural portrait of Canada Highlight tables, 2006 Census. 97-562-XWE2006002. Ottawa, Statistics Canada.

22. Canadian Diabetes Association Clinical Practice Guidelines Expert Committee (2008) Canadian Diabetes Association 2008 clinical practice guidelines for the prevention and management of diabetes in Canada. Can J Diabetes 32:S1-S201

23. Hux JE, Ivis F, Flintoft V, Bica A (2002) Diabetes in Ontario: determination of prevalence and incidence using a validated administrative data algorithm. Diabetes Care 25:512-516

24. Deyo RA, Cherkin DC, Ciol MA (1992) Adapting a clinical comorbidity index for use with ICD-9-CM administrative databases. J Clin Epidemiol 45:613-619

25. Shah BR, Chiu M, Amin S, Ramani M, Sadry S, Tu JV (2010) Surname lists to identify South Asian and Chinese ethnicity from secondary data in Ontario, Canada: a validation study. BMC Med Res Methodol 10:42

26. Leiter LA, Barr A, Bélanger A et al (2001) Diabetes screening in Canada (DIASCAN) study. Diabetes Care 24:1038-1043

27. Anna V, van der Ploeg HP, Cheung NW, Huxley RR, Bauman AE (2008) Sociodemographic correlates of the increasing trend in prevalence of gestational diabetes mellitus in a large population of women between 1995 and 2005. Diabetes Care 31:2288-2293

28. Savitz DA, Janevic TM, Engel SM, Kaufman JS, Herring AH (2008) Ethnicity and gestational diabetes in New York City, 1995-2003. BJOG 115:969-978

29. Bellamy L, Casas JP, Hingorani AD, Williams D (2009) Type 2 diabetes mellitus after gestational diabetes: a systematic review and meta-analysis. Lancet 373:1773-1779

30. Retnakaran R, Hanley AJG, Connelly PW, Sermer M, Zinman B (2006) Ethnicity modifies the effect of obesity on insulin resistance in pregnancy: a comparison of Asian, South Asian, and Caucasian women. J Clin Endocrinol Metab 91:93-97

31. O'Sullivan JB, Mahan CM (1964) Criteria for the oral glucose tolerance test in pregnancy. Diabetes 13:278-285

32. Snyder J, Shrim A, Nudi M, Meltzer SJ (2010) Ethnic differences in gestational diabetes testing results, prevalence and perinatal outcomes. Can J Diabetes 34:250

33. Wilson S, Rosella L, Lipscombe L, Manuel D (2010) The effectiveness and efficiency of diabetes screening in Ontario, Canada: a population-based cohort study. BMC Publ Health 10:506

34. Ng E, Dasgupta K, Johnson JA (2008) An algorithm to differentiate diabetic respondents in the Canadian Community Health Survey. Health Reports 19:71-79

35. Maddigan SL, Feeny DH, Majumdar SR, Farris KB, Johnson JA (2006) Understanding the determinants of health for people with type 2 diabetes. Am J Public Health 96:1649-1655

36. Brar S, Tang S, Drummond N et al (2009) Perinatal care for South Asian immigrant women and women born in Canada: telephone survey of users. J Obstet Gynaecol Can 31:708-716

37. Alberti KGMM, Zimmet P, Shaw J (2006) Metabolic syndrome: a new world-wide definition. A Consensus Statement from the International Diabetes Federation. Diabet Med 23:469-480

38. WHO expert consultation (2004) Appropriate body-mass index for Asian populations and its implications for policy and intervention strategies. Lancet 363:157-163

39. Razak F, Anand SS, Shannon H et al (2007) Defining obesity cut points in a multiethnic population. Circulation 115:2111-2118 